\title{
Erythrocyte Shape Abnormalities, Membrane Oxidative Damage, and $\beta$-Actin Alterations: An Unrecognized Triad in Classical Autism
}

\author{
Lucia Ciccoli, ${ }^{1}$ Claudio De Felice, ${ }^{2}$ Eugenio Paccagnini, ${ }^{3}$ Silvia Leoncini, ${ }^{1,4}$ \\ Alessandra Pecorelli, ${ }^{1,4}$ Cinzia Signorini, ${ }^{1}$ Giuseppe Belmonte, ${ }^{5}$ Roberto Guerranti, ${ }^{6,7}$ \\ Alessio Cortelazzo, ${ }^{4,7}$ Mariangela Gentile, ${ }^{3}$ Gloria Zollo, ${ }^{1,4}$ Thierry Durand, ${ }^{8}$ \\ Giuseppe Valacchi, ${ }^{9,10}$ Marcello Rossi, ${ }^{11}$ and Joussef Hayek ${ }^{4}$ \\ ${ }^{1}$ Department of Molecular and Developmental Medicine, University of Siena, Via A. Moro 2, 53100 Siena, Italy \\ ${ }^{2}$ Neonatal Intensive Care Unit, University Hospital, Azienda Ospedaliera Universitaria Senese (AOUS), \\ Viale M. Bracci 16, 53100 Siena, Italy \\ ${ }^{3}$ Department of Life Sciences, University of Siena, Via A. Moro 2, 53100 Siena, Italy \\ ${ }^{4}$ Child Neuropsychiatry Unit, University Hospital, AOUS, Viale M. Bracci 16, 53100 Siena, Italy \\ ${ }^{5}$ Medicine, Surgery and Neurosciences Department, University of Siena, Viale M. Bracci 16, 53100 Siena, Italy \\ ${ }^{6}$ Clinical Pathology Laboratory Unit, University Hospital, AOUS, Viale M. Bracci 16, 53100 Siena, Italy \\ ${ }^{7}$ Department of Medical Biotechnologies, University of Siena, Via A. Moro 2, 53100 Siena, Italy \\ ${ }^{8}$ Institut des Biomolécules Max Mousseron (IBMM), UMR 5247, CNRS/UM1/UM2, BP 14491 34093, Montpellier, Cedex 5, France \\ ${ }^{9}$ Life Science and Biotechnologies, University of Ferrara, Via Borsari 46, 44100 Ferrara, Italy \\ ${ }^{10}$ Department of Food and Nutrition, Kyung Hee University, 1 Hoegi-dong, Dongdaemun-gu, Seoul 130-701, Republic of Korea \\ ${ }^{11}$ Respiratory Pathophysiology and Rehabilitation Unit, University Hospital, AOUS, Viale M. Bracci 16, 53100 Siena, Italy
}

Correspondence should be addressed to Claudio De Felice; geniente@gmail.com

Received 2 October 2013; Accepted 22 October 2013

Academic Editor: Paul Ashwood

Copyright (c) 2013 Lucia Ciccoli et al. This is an open access article distributed under the Creative Commons Attribution License, which permits unrestricted use, distribution, and reproduction in any medium, provided the original work is properly cited.

Autism spectrum disorders (ASDs) are a complex group of neurodevelopment disorders steadily rising in frequency and treatment refractory, where the search for biological markers is of paramount importance. Although red blood cells (RBCs) membrane lipidomics and rheological variables have been reported to be altered, with some suggestions indicating an increased lipid peroxidation in the erythrocyte membrane, to date no information exists on how the oxidative membrane damage may affect cytoskeletal membrane proteins and, ultimately, RBCs shape in autism. Here, we investigated RBC morphology by scanning electron microscopy in patients with classical autism, that is, the predominant ASDs phenotype (age range: 6-26 years), nonautistic neurodevelopmental disorders (i.e., "positive controls"), and healthy controls (i.e., "negative controls"). A high percentage of altered RBCs shapes, predominantly elliptocytes, was observed in autistic patients, but not in both control groups. The RBCs altered morphology in autistic subjects was related to increased erythrocyte membrane $\mathrm{F}_{2}$-isoprostanes and 4-hydroxynonenal protein adducts. In addition, an oxidative damage of the erythrocyte membrane $\beta$-actin protein was evidenced. Therefore, the combination of erythrocyte shape abnormalities, erythrocyte membrane oxidative damage, and $\beta$-actin alterations constitutes a previously unrecognized triad in classical autism and provides new biological markers in the diagnostic workup of ASDs.

\section{Introduction}

Autism spectrum disorders (ASDs) are considered to be the result of a complex interaction between a genetic background and environmental factors $[1,2]$. Autism is a heterogeneous, behaviorally defined neurodevelopmental disorder affecting four times more males than females [3], with a clinical onset usually within the 2 nd year of life and mainly consisting of social impairment; communication difficulties; and restricted, repetitive, and stereotyped patterns of behavior. 
Classical autism is the overwhelmingly predominant ASDs phenotype, which also include other four disorders, that is, Asperger syndrome and pervasive developmental disorder not otherwise specified (PDD-NOS), and childhood disintegrative disorder. Rett syndrome (RTT) is a genetically determined neurodevelopmental disorder with autistic features, and has been recently separated by the ASDs as a distinct nosological entity (Diagnostic and Statistical Manual of mental disorders V-DSMV).

The search for specific and reliable biomarkers is of paramount importance in autism, given its dramatically rising prevalence in the general population over the last two decades [4] from 1 in 5000 in the mid-1970s to 1 in 88 in 2008 [4, 5].

A redox imbalance has been repeatedly reported in autism [6-8] although its role in the pathogenesis is still under question. In particular, it is unclear whether oxidative stress (OS) is a cause or consequence of autism [9]. Recent studies indicate that autism is associated with deficits in glutathione antioxidant defense in selective regions of the brain, thus potentially contributing to OS, immune dysfunction, and apoptosis, particularly in the cerebellum and temporal lobe [10].

More recently, OS and erythrocyte membrane alterations have been described in autistic children, including elevated erythrocyte thiobarbituric acid reactive substances, urinary isoprostane, and hexanoyl-lysine levels, associated with a significant reduction of $\mathrm{Na}^{+} / \mathrm{K}^{+}$-ATPase activity, a reduction of the erythrocyte membrane fluidity and an alteration in the erythrocyte fatty acid membrane profile linked to an increase in monounsaturated fatty acids, a decrease in eicosapentaenoic acid and docosahexaenoic acid, and a consequently increased $\omega-6 / \omega-3$ ratio [11].

The peculiar triad of OS imbalance, mild chronic hypoxia, and an abnormally high frequency of leptocytes in the peripheral blood has been reported by our group in girls RTT [12], a relatively rare neurodevelopmental disorder almost affecting females and mainly due to de novo mutations of the X-linked methyl-CpG-binding protein $2(\mathrm{MeCP} 2)$ gene [13]. These data indicate that redox imbalance and oxygen exchange could be key players in the pathogenesis of this particular human model of autism.

The shape is critical to red blood cells (RBCs) function and blood rheological properties, and emerging evidence indicates that OS is a key factors in modulating erythrocyte shape [14-16]. Although RBCs membrane lipidomics [11] and rheological variables [17] have been reported to be altered, and some suggestions of an increased lipid peroxidation in the erythrocyte membrane exist [11], to date no information is available on how the RBCs oxidative membrane damage may affect cytoskeletal membrane proteins and, ultimately, erythrocyte shape in autism.

In the present study, we investigated RBC morphology, in situ membrane oxidative damage, and cytoskeletal proteins in patients with classic autistic disorder.

\section{Methods}

2.1. Subjects Population. A total of $n=15$ patients (male: 9; female: 6), with classic autistic disorder (mean age at examination: $15.9 \pm 5.9$ years, range 6-26), as well as $n=15$ healthy controls of comparable age and a typical neurodevelopment (mean age: $16.3 \pm 6.2$ years, range $5-30$; male: 8; female: 7) participated in the study. Furthermore, a third group of 15 patients (male: 7; female: 8) with nonautistic neurodevelopmental disorders (NA-NDDs) (mean age at examination: $15.8 \pm 6.0$ years, range $5-28$ ) was enrolled as a "positive control" population, including idiopathic mental retardation $(n=6)$, cerebral palsy $(n=3)$, Attention-Deficit/ Hyperactivity Disorder (ADHD) $(n=4)$, and language disorders $(n=2)$. Childhood Autism Rating Scale scores (CARS) [18] for the examined autistic patients and the NANDDs groups were estimated. The populations of patients and healthy subjects were recruited by the medical staff of the Child Neuropsychiatry Unit of the Azienda Ospedaliera Senese (Siena, Italy). All the enrolled patients and healthy subjects were genetically unrelated. The autistic patients were diagnosed by DSMV and evaluated using Autism Diagnostic Observation Schedule (ADOS), and Autism Behaviour Checklist (ABC). Patients with RTT, X-fragile syndrome, or tuberous sclerosis, as well as subjects with clinical evident sideropenic anemia, perinatal adverse events, and/or brain abnormalities on magnetic resonance imaging, were excluded for the present study. All subjects were on a typical Mediterranean diet.

The study was conducted after the approval by the Institutional Review Board and all written informed consents were obtained from either the parents or the legal tutors of the enrolled patients.

2.2. Routine Hematological Analyses. For these particular laboratory determinations, samples collected in tubes with $\mathrm{K}_{2}$ EDTA were analyzed by an automated hematology system Sysmex XE-2100 (Sysmex corporation, Japan) in the automated aspiration (i.e., closed) sampling mode, using $200 \mu \mathrm{L}$ sample volume. The instruments are in routine use for count blood cells and automated differential counts analyses and underwent periodic quality assessment in internal and external control programs. A 5-part differential count was performed by lysing erythrocytes and analyzing the light scatter/fluorescence [19]. Blood smears were stained with standard May-Grünwald Giemsa within 6 hours after blood sampling (SP1000 instrument) and visualized by an automated image recognition system CellaVision DM96, an automated microscope with software showing digitalized images of the blood smears.

\subsection{Blood Sampling for Erythrocyte Oxidative and Shape} Analysis Studies. For these aims, an aliquot of blood was collected in heparinized tubes, and manipulations were carried out within 2 hrs after sample collection. Blood samples were centrifuged at $2400 \times \mathrm{g}$ for $15 \mathrm{~min}$ at $4^{\circ} \mathrm{C}$, whereas the platelet poor plasma and the buffy coat were removed by aspiration. RBCs were washed twice with physiological solution $(150 \mathrm{mM} \mathrm{NaCl})$. An aliquot of packed erythrocytes was resuspended in Ringer solution (125 mM NaCl, $5 \mathrm{mM}$ $\mathrm{KCl}, 1 \mathrm{mM} \mathrm{MgSO}$, $32 \mathrm{mM} \mathrm{N}$-2-hydroxyethylpiperazine-N2-ethanesulfonic acid (HEPES), $5 \mathrm{mM}$ glucose, $1 \mathrm{mM} \mathrm{CaCl}$ ), $\mathrm{pH} 7.4$ as a $50 \%(\mathrm{vol} / \mathrm{vol})$ suspension for the determination 
of intraerythrocyte nonprotein-bound-iron (NPBI). The remaining volume of packed RBCs was used for erythrocyte membranes preparations (i.e., hemoglobin-free ghosts) for 4-hydroxynonenal protein adducts (4-HNE PAs) determinations.

An aliquot of each blood sample $(1 \mathrm{~mL})$ was centrifuged at $800 \times \mathrm{g}$ for 10 minutes at $4^{\circ} \mathrm{C}$ for scanning electron microscopy (SEM) analysis of erythrocytes.

2.4. Scanning Electron Microscopy (SEM). As previously described [12], erythrocytes were plated on poly-l-lysine coated slides and fixed in Karnowsky (2.5\% glutaraldehyde$4 \%$ paraformaldehyde in $0.1 \mathrm{M}$ sodium-cacodylate buffer, $\mathrm{pH}$ 7.2) for $2 \mathrm{~h}$ at $4^{\circ} \mathrm{C}$, rinsed twice for $10 \mathrm{~min}$ with $0.1 \mathrm{M}$ sodium cacodylate buffer and postfixed in $1 \%$ osmium tetroxide in $0.1 \mathrm{M}$ sodium-cacodylate buffer for $1 \mathrm{~h}$ at $4^{\circ} \mathrm{C}$. Specimens were then dehydrated through a graded ethanol series, dried in a $\mathrm{CO}_{2}$ critical point dryer (CPD010, Balzers Union, Liechtenstein), mounted on specimen stub, sputter coated with gold (Sputter Coater S150B, Edwards, England), and examined in a XL 20 SEM (Philips, Eindhoven, Netherlands). Altered RBCs shapes were evaluated by counting $\geq 800$ cells (50 erythrocytes for each different SEM field at a magnification of $\times 3000$ ) from all groups of subjects. All countings were carried out in triplicate and averages were taken for data analysis.

2.5. Intraerythrocyte NPBI. Intraerythrocyte NPBI (nmol/ $\mathrm{mL}$ ) was determined as a desferrioxamine (DFO)-iron complex (ferrioxamine), as previously reported [20]. Briefly, $25 \mu \mathrm{M}$ DFO was added to the samples $(1 \mathrm{~mL}$ erythrocyte suspension), the erythrocytes were then lysed by adding water $(1 \mathrm{vol})$ and freezing $\left(-70^{\circ} \mathrm{C}\right)$ and thawing. The hemolysate was ultrafiltered at $3373 \times \mathrm{g}$ for $30 \mathrm{~min}$ in centrifugal filters with a $30 \mathrm{kDa}$ molecular weight cutoff (VIVASPIN 4, Sartorius Stedim Biotech $\mathrm{GmbH}$, Goettingen Germany) and the ultrafiltrate was stored at $-20^{\circ} \mathrm{C}$ until analysis. The DFO excess was removed by silica (Silicagel; 25-40 $\mu \mathrm{m}$ ) column chromatography. The DFO-iron complex was determined by HPLC and the detection wavelength was $229 \mathrm{~nm}$. The calibration curve correlation for intraerythrocyte NPBI was adequate $\left(r^{2}=0.994009\right)$, the minimum detection limit was $0.1 \mathrm{nmol} / \mathrm{mL}$, and mean intra- and inter-observer coefficients of variation were $\leq 2.5 \%$ and $\leq 5 \%$, respectively.

2.6. Erythrocyte Membrane Preparation. An aliquot $(600 \mu \mathrm{L})$ of packed RBCs was lysed in Dodge buffer, and erythrocyte membranes were prepared, according to Dodge et al. [21], by repeated washing until the "ghosts" were pearly white. Samples were kept frozen at $-70^{\circ} \mathrm{C}$ until used for sodium dodecyl sulfate-polyacrylamide gel electrophoresis (SDS-PAGE), immunoprecipitation (IP), and esterified $\mathrm{F}_{2}$ isoprostanes ( $\mathrm{F}_{2}$-IsoPs).

2.7. Immunoprecipitation of Erythrocyte $\beta$-Actin. Erythrocyte membrane (ghosts) proteins $(200 \mu \mathrm{g})$ were incubated with $5 \mu \mathrm{g} \beta$-actin antibody (Millipore Corporation, Billerica, MA, USA) overnight at $4^{\circ} \mathrm{C}$ on a rotator. Then, immune complex was incubated with $50 \mu \mathrm{L}$ of Protein A-Sepharose (SigmaAldrich, Milan, Italy) and rotated at $4^{\circ} \mathrm{C}$ for $2 \mathrm{~h}$. Samples were centrifuged at 10,000 $\mathrm{g}$ for $5 \mathrm{~min}$ and washed three times with $1 \mathrm{~mL}$ ice-cold PBS. The pellet was mixed with $2 \mathrm{X}$ reducing sample buffer, boiled, and loaded on SDS-PAGE gels for silver staining or western blotting analysis.

2.8. Silver Staining of Erythrocyte Membrane Proteins and Immunoprecipitated $\beta$-Actin. Erythrocyte membrane (i.e., "ghosts") proteins (20 $\mu$ g protein, determined using BioRad protein assay; BioRad, Hercules, CA) or immunoprecipitated $\beta$-actin were separated by the polyacrylamide gel electrophoresis (one-dimensional) method for discontinuous SDS-PAGE on $10 \%$ polyacrylamide gels in denaturing conditions, according to Laemmli [22]. At the end of the electrophoretic run, gels were stained with silver nitrate for protein visualization (Sigma-Aldrich, Milan, Italy). Gel image was acquired using image scanner and the bands were automatically detected and analyzed using TotalLab software (nonlinear dynamics, version 1.0). Band volume was expressed as a ratio of the total protein volume detected from the entire gel to minimize differences between band (band normalization) and to compare band measurements in different lanes.

2.9. Western Blot for 4-HNE Protein Adducts in Erythrocyte Membrane Proteins and Immunoprecipitated $\beta$-Actin. Western blot protocols were performed as previously described [12]. Erythrocyte membrane proteins ( $40 \mu \mathrm{g}$ protein, determined using BioRad protein assay; BioRad, Hercules, CA) or the immunoprecipitated $\beta$-actin were resolved on $10 \%$ SDSPAGE gels and transferred onto a hybond ECL nitrocellulose membrane (GE Healthcare Europe GmbH, Milan, Italy). After blocking in 3\% nonfat milk (BioRad, Hercules, CA, USA), the membranes were incubated overnight at $4^{\circ} \mathrm{C}$ with goat polyclonal anti-4-HNE adduct antibody (cod. AB5605; Millipore Corporation, Billerica, MA, USA). Following washes in TBS Tween and incubation with specific secondary antibody (mouse anti-goat horseradish peroxidaseconjugated, Santa Cruz Biotechnology, Inc., CA, USA) for $1 \mathrm{~h}$ at RT, the membranes were incubated with ECL reagents (BioRad, Hercules, CA, USA) for $1 \mathrm{~min}$. Images were digitized (ChemiDoc XRS, BioRad, Hercules, CA) and band optical densities were quantified using a computerized imaging system (Quantity One Imaging system).

2.10. Erythrocyte Membrane Esterified $F_{2}$-Isoprostanes $\left(F_{2}-\right.$ IsoPs). A $100 \mu \mathrm{L}$ aliquot of the erythrocyte membrane samples was resuspended with $\mathrm{H}_{2} \mathrm{O}(0.9 \mathrm{~mL})$ and $1 \mathrm{~N} \mathrm{KOH}$ $(500 \mu \mathrm{L})$ was added for the basic hydrolysis. After incubation at $45^{\circ} \mathrm{C}$ for $45 \mathrm{~min}$, the sample was acidified to $\mathrm{pH} 3$ with $\mathrm{HCl} 1 \mathrm{~N}(500 \mu \mathrm{L})$, spiked with tetradeuterated Prostaglandin $\mathrm{F}_{2 \alpha}\left(\mathrm{PGF}_{2 \alpha}-\mathrm{d}_{4}\right)(500 \mathrm{pg}$ in $50 \mu \mathrm{L}$ of ethanol; Cayman, Ann Arbor, MI, USA), as internal standard, and extracted with $10 \mathrm{~mL}$ of ethyl acetate. The upper organic layer, obtained after centrifugation at $1000 \times \mathrm{g}$ for $5 \mathrm{~min}$, was applied onto an aminopropyl $\left(\mathrm{NH}_{2}\right)$ cartridge (Waters, Milford, MA, U.S.A.) preconditioned with $10 \mathrm{~mL}$ of hexane. After derivatization the determination of $\mathrm{F}_{2}$-IsoPs was accomplished by gas chromatography/negative ion chemical ionization tandem mass spectrometry (GC/NICI-MS/MS) analysis [12]. 
Esterified $\mathrm{F}_{2}$-IsoPs were normalized for membrane proteins and quantified by BioRad protein assay (BioRad, Hercules, CA) using $0.2 \%$ Triton X-100 to dissolve the membranes. The measured ions were the product ions at $\mathrm{m} / z$ 299 and $m / z 303$ derived from the [M-181] $]^{-}$precursor ions $(\mathrm{m} / z 569$ and $m / z 573)$ produced from $15-\mathrm{F}_{2}$ t-IsoPs (the most represented $\mathrm{F}_{2}$-IsoP isomer) and $\mathrm{PGF}_{2 \alpha}-\mathrm{d}_{4}$, respectively [12].

2.11. Statistical Data Analysis. All variables were tested for normal distribution (D'Agostino-Pearson test) and data were presented as means \pm SD for normally distributed variables. Differences between groups were evaluated using independent-sample $t$-test (continuous normally distributed data), Mann-Whitney rank sum test (continuous nonnormally distributed data), chi-square statistics (categorical variables with minimum number of cases per cell $\geq 5$ ) or Fisher's exact test (categorical variables with minimum number of cases per cell $<5$ ), one-way analysis of variance (ANOVA), Student-Newman-Keuls post hoc test or Kruskal-Wallis test. Associations between variables were tested by univariate regression analysis (Pearson's coefficients or Spearman's rho, as appropriate). The effects of small population sizes on possible type I $(\alpha)$ /type II $(\beta)$ errors in the data interpretation were examined using a sampling size algorithm. A two-sided $P<0.05$ was considered to indicate statistical significance, and the Bonferroni-corrected significance levels were used for multiple $t$-tests. The MedCalc version 12.1.4 statistical software package (MedCalc Software, Mariakerke, Belgium) was used.

\section{Results}

3.1. IQ and CARS Estimates. Estimated IQ and CARS values for the autistic group were $40.6 \pm 12.80$ (range 20-60), and $51.9 \pm 7.0$ (range 41-60), respectively, versus $63.0 \pm 8.54$ (values range 55-72) and $26.0 \pm 2.2$ (values range: $24-29$ ), respectively, ( $t$-test statistics 5.638 and -13.671 , respectively; $P$ value $s<0.0001)$.

3.2. Red Blood Cell Counts. Blood cell counts in patients were not significantly different from those of the control groups, with the single exception of a nonsignificant trend for MCHC, slightly lower in the autistic group ( ${ }^{*} P \geq 0.0669$ ) (Table 1). In particular, no laboratory signs of anemia in any of the three groups were evidenced. Nevertheless, statistical differences regarding $\mathrm{Hb}$ and $\mathrm{MCV}$ were detectable for the NA-NDDs group (Table 1). These findings would suggest a "relative microcytic anemia" in this latter population.

At the SEM analysis, significantly higher percentages of altered RBCs shapes were present in the patients' groups, as compared to healthy control subjects (Figures 1 and 2). In particular, abnormally shaped erythrocytes in the blood samples from autistic subjects predominantly featured elliptocytes (33.2 $\pm 11.2 \%$ of all erythrocytes, range: $15.4-55.5)$. Besides elliptocytes, various abnormal RBC shape were observed, including echinocytes, leptocytes, knizocytes, codocytes, and stomatocytes.

The NA-NDDs group showed mixed abnormally shaped RBCs, without an overwhelmingly predominant erythrocyte
TABLE 1: Routine RBC variables in subjects with classical autism and nonautistic neurodevelopmental disorders (NA-NDDs) versus healthy controls.

\begin{tabular}{lcccc}
\hline RBC variables & $\begin{array}{c}\text { Healthy } \\
\text { controls }\end{array}$ & NA-NDDs & $\begin{array}{c}\text { Classical } \\
\text { autism }\end{array}$ & $\begin{array}{c}P \text { value } \\
(\text { ANOVA) }\end{array}$ \\
\hline $\mathrm{Hb}(\mathrm{g} / \mathrm{dL})$ & $14.5 \pm 0.9$ & $13.6 \pm 1.0^{*}$ & $14.2 \pm 1.6$ & $\mathbf{0 . 0 0 1}$ \\
$\mathrm{MCV}(\mathrm{fL})$ & $85.1 \pm 6.6$ & $83.3 \pm 4.4^{*}$ & $86.1 \pm 4.0$ & $\mathbf{0 . 0 0 4}$ \\
$\mathrm{MCH}(\mathrm{pg} / \mathrm{cell})$ & $28.8 \pm 2.4$ & $27.9 \pm 1.6$ & $28.6 \pm 1.9$ & 0.362 \\
$\mathrm{MCHC}(\mathrm{g} / \mathrm{dL})$ & $33.9 \pm 1.0$ & $33.6 \pm 0.9$ & $33.2 \pm 0.9$ & 0.490 \\
$\mathrm{RDW}(\%)$ & $13.4 \pm 1.5$ & $14.0 \pm 2.1$ & $13.1 \pm 0.7$ & 0.188 \\
\hline
\end{tabular}

Data are expressed as means \pm SD. ${ }^{*} P$ value $<0.05$ (post hoc analysis). Bold character indicates statistically significant differences.

$\mathrm{Hb}$ : hemoglobin; MCV: mean corpuscular volume; $\mathrm{MCH}$ : mean corpuscular hemoglobin; MCHC: mean corpuscular hemoglobin concentration; RDW: red cell distribution width.

shape phenotype, although a slight prevalence of leptocytes ( 25\%) was detectable.

Significant positive relationships were observed for the percentages of discocytes, leptocytes, or stomatocytes and RDW ( $\rho$ correlation coefficients range: 0.7245 to 0.8915 ). In addition, significant positive relationship were evidenced for the percentages of knizocytes and stomatocytes with $\mathrm{Hb}(\rho=$ 0.6907 and $\rho=0.5855$, resp.), and the percentage of stomatocytes versus MCHC $(\rho=0.6070)$. Significant inverse relationships were found between percentages of codocytes and RDW $(\rho=-0.8181)$, percentage of echinocytes and MCHC $(\rho=-0.8379)$, and echinocytes versus $\mathrm{Hb}(\rho=-0.7776)$ (Table 2).

3.3. Erythrocyte Oxidative Status. Intraerythrocyte NPBI, together with erythrocyte membrane esterified $\mathrm{F}_{2}$-IsoPs and 4-HNE PAs, were significantly increased in autistic subjects, as well as patients with NA-NDDs ( $F$-ratio range: 6.41 to $64.31 ; P$ value range: 0.001 to 0.004$)$, as compared to healthy controls (Figure 3). A distinct 4-HNE PAs pattern was detectable (i.e., autism $>$ NA-NDDs $>$ healthy controls), thus indicating that classical autism and NA-NDDs are sharing erythrocyte oxidative membrane damage, although membrane proteins likely undergo different degrees of oxidation.

3.4. Erythrocyte Membrane Proteins. Typical SDS-PAGE analysis are shown in Figure 4(a). Alterations in the whole electrophoretic pattern of autistic patients were observed. In particular, a quantitative decrease in $\beta$-actin $(-16$ to $-18.8 \%$; $F$-ratio $=314.5 ; P<0.001)$ was evidenced and further confirmed in the western blot analysis carried out after actinIP by using a specific antibody (Figure 4(b)). In autistic patients the blotting study indicated detectable protein bands showing increased binding with 4 -HNE, that is, the major aldehyde product of lipid peroxidation, as compared to both negative and positive control subjects (Figure $4(\mathrm{c})$ ). $\beta$-actin was found to be a major target for 4-HNE binding, as indicated by densitometric analysis $(+61.9$ to $+78.8 \%$; $F$-ratio $=2622.8 ; P<0.001$ ) following $\beta$-actin IP (Figure $4(\mathrm{~d})$ ).

3.5. Correlations between Erythrocyte Shape, Membrane Oxidative Markers and $\beta$-Actin. The percentage of elliptocytes 


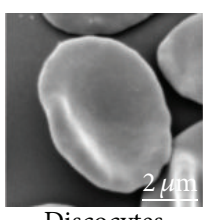

Discocytes

(a)

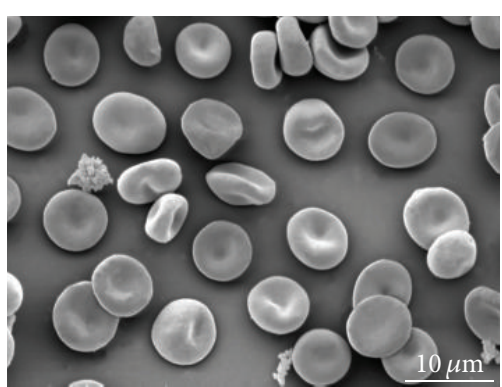

(h)

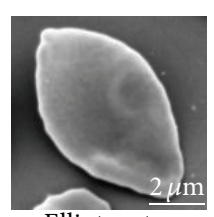

Elliptocytes

(b)

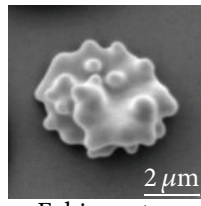

Echinocytes

(c)

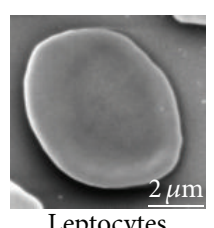

(d)

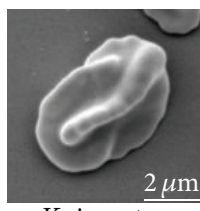

Knizocytes

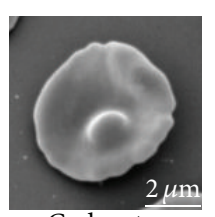

Codocytes

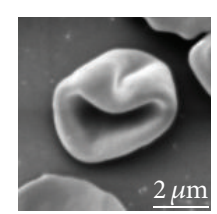

Stomatocytes

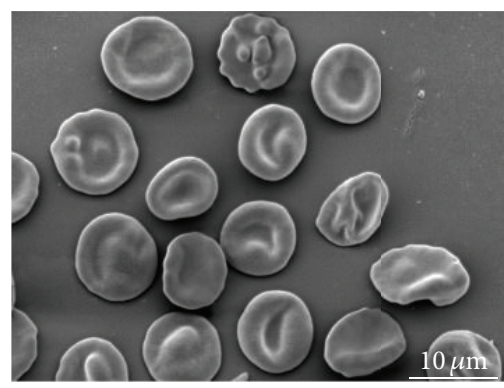

(i) (f)

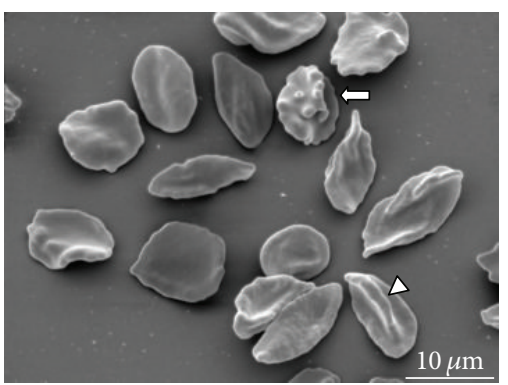

(j)

FIGURE 1: Abnormal erythrocyte shapes in classical autism at the scanning electron microscopy (SEM). (a): normal discocyte shape; (b) to (g): main shape-altered RBCs observed in autistic patients; (h): healthy controls; (i): a typical morphological pattern in nonautistic neurodevelopmental disorders (NA-NDDs); (j): typical picture in an autistic patient with predominant elliptocytosis. Symbols indicate intermediate-shaped RBCs: the arrow indicates a disco-echinocyte shape, while the arrowhead indicates the presence of a knizo-echinocyte shape in autistic patients, bars correspond to $2 \mu \mathrm{m}$ in (a) to (g) upper panels and to $10 \mu \mathrm{m}$ in the (h), (i), and (j) lower panels.

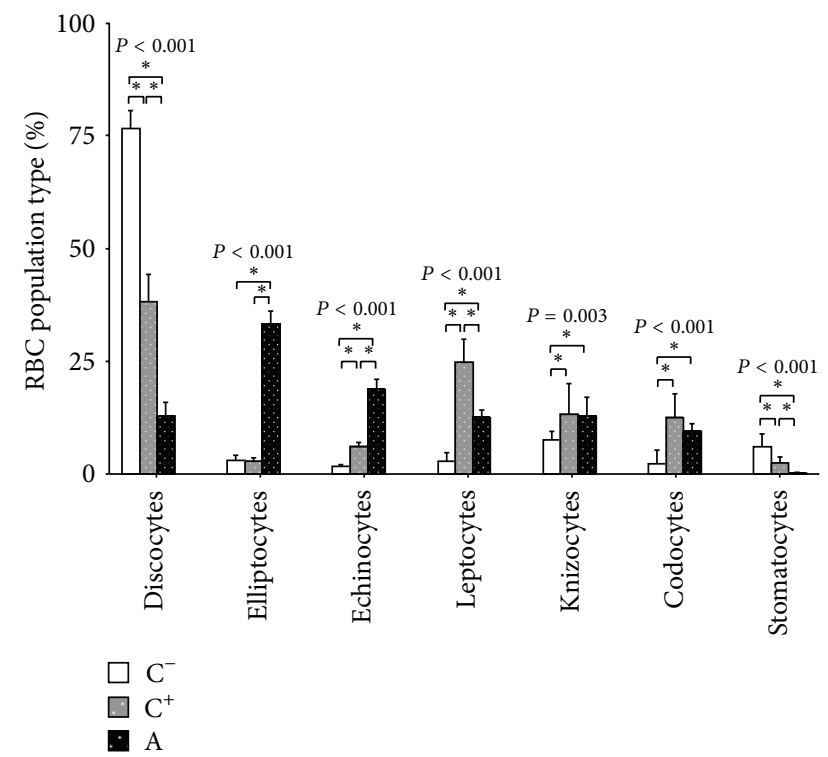

FIGURE 2: RBC morphology distribution in autistic patients, subjects with nonautistic neurodevelopmental disorders (NA-NDDs) and healthy controls. Statistically significant differences are denoted by single asterisk, $P>0.05$. $P$ values above each erythrocyte shape classification refer to one-way ANOVA.

was positively correlated with IE-NPBI, erythrocyte membrane 4-HNE PAs, and esterified $\mathrm{F}_{2}$-IsoPs, as well as membrane 4 -HNE $\beta$-actin adducts (4-HNE $\beta$-AAs) levels. The examined OS markers showed positive associations between them, while elliptocytes were inversely related to $\beta$-actin membrane content, and a remarkably high inverse relationship between amount of $\beta$-actin in the membrane and 4 -HNE $\beta$-AAs was observed $(r=-0.960 ; P<0.001)$ (Table 3 ).

\section{Discussion}

Our findings show the coexistence of a peculiar combination of erythrocyte shape abnormalities, erythrocyte membrane oxidative damage, and $\beta$-actin alterations, which represents a previously unrecognized triad in classical autism.

4.1. RBCs Morphology. Several conditions, either congenital or acquired, are known to lead to abnormal erythrocyte shape [23]. The maintenance of the discoid shape is of paramount importance for the RBC main physiological role (i.e., transport of respiratory gases to and from tissues), while the deformability of the circulating cells has critical effects on the rheological properties of blood [24].

Our observations indicate that elliptocytes are the predominant abnormal erythrocyte shape occurring in the peripheral blood from patients with classical autism, and, to the best of our knowledge, this is the first time that an abnormal erythrocyte shape is reported in ASDs other than RTT. The presence of abnormal RBC shapes appears to be associated with an altered redox status in erythrocytes and a decrease and/or oxidization for some of the main cytoskeletal proteins known to be critical for the maintenance of the horizontal interactions in the erythrocyte membrane architecture. Although, abnormal RBC shape are reported in a series of associated conditions [23], this is likely the first time that an elliptocytosis is described outside of the known conditions of hereditary elliptocytosis and thalassemias [25]. Hereditary elliptocytosis is caused by weakened horizontal 
TABLE 2: Correlation matrix for RBC shape as a function of RBC parameters in autistic patients.

\begin{tabular}{|c|c|c|c|c|c|c|c|}
\hline \multirow{2}{*}{$\mathrm{RBC}$ variables } & \multicolumn{7}{|c|}{ Erythrocyte shape class } \\
\hline & Disco- & Ellipto- & Echino- & Lepto- & Knizo- & Codo- & Stomato- \\
\hline \multirow{2}{*}{$\mathrm{Hb}$} & 0.0159 & 0.1831 & $-0.7776^{*}$ & 0.1151 & $0.6907^{*}$ & -0.0336 & $0.5855^{*}$ \\
\hline & $(0.5452)$ & $(0.9729)$ & $(0.0011)$ & $(0.4698)$ & $(0.0062)$ & $(0.9864)$ & $(0.0278)$ \\
\hline \multirow{2}{*}{$\mathrm{MCV}$} & -0.2616 & 0.2063 & -0.0556 & -0.2841 & 0.2308 & 0.1960 & 0.4090 \\
\hline & $(0.1479)$ & $(0.2834)$ & $(0.8471)$ & $(0.2442)$ & $(0.3414)$ & $(0.4069)$ & $(0.1580)$ \\
\hline \multirow{2}{*}{$\mathrm{MCH}$} & -0.0771 & 0.2980 & -0.3959 & -0.1329 & 0.2868 & 0.0847 & 0.4720 \\
\hline & $(0.6423)$ & $(0.3586)$ & $(0.1732)$ & $(0.4530)$ & $(0.1144)$ & $(0.8030)$ & $(0.1099)$ \\
\hline \multirow{2}{*}{$\mathrm{MCHC}$} & 0.2571 & 0.3688 & $-0.8379^{*}$ & -0.0232 & 0.2720 & -0.1233 & $0.6070 *$ \\
\hline & $(0.3502)$ & $(0.1553)$ & $(0.0002)$ & $(0.8842)$ & $(0.2860)$ & $(0.3714)$ & $(0.0214)$ \\
\hline \multirow{2}{*}{ RDW } & $0.8915^{*}$ & -0.0476 & -0.4498 & $0.7245^{*}$ & 0.0357 & $-0.8181^{*}$ & $0.8278^{*}$ \\
\hline & $(0.0050)$ & $(0.8806)$ & $(0.0672)$ & $(0.0078)$ & $(0.9516)$ & $(0.0038)$ & $(0.0031)$ \\
\hline
\end{tabular}

Data are Spearman's rho correlation coefficients with in brackets $P$ values $(N=15)$. Bold characters with asterisks indicate statistically significant correlations. Disco-: discocytes; Ellipto-: elliptocytes; Echino-: echinocytes; Lepto-: leptocytes; Knizo-: knizocytes; Codo-: codocytes; Stomato-: stomatocytes; Hb: hemoglobin; MCV: mean corpuscular volume; MCH: mean corpuscular hemoglobin; MCHC: mean corpuscular hemoglobin concentration; RDW: red cell distribution width.

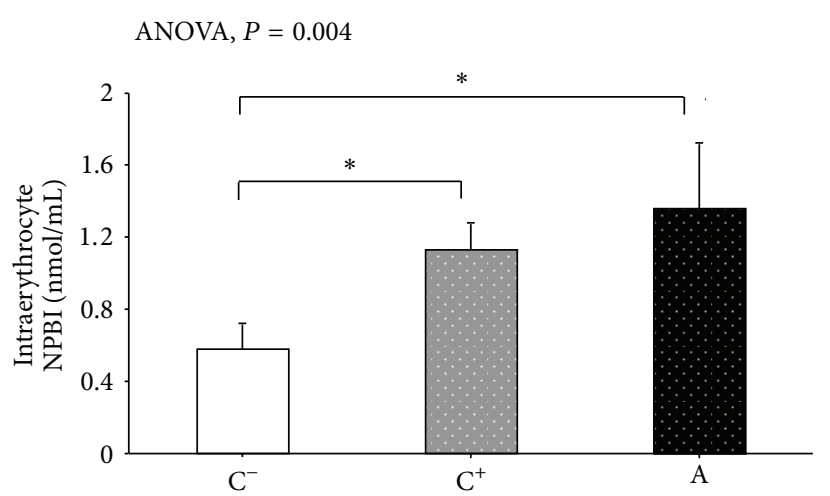

(a)

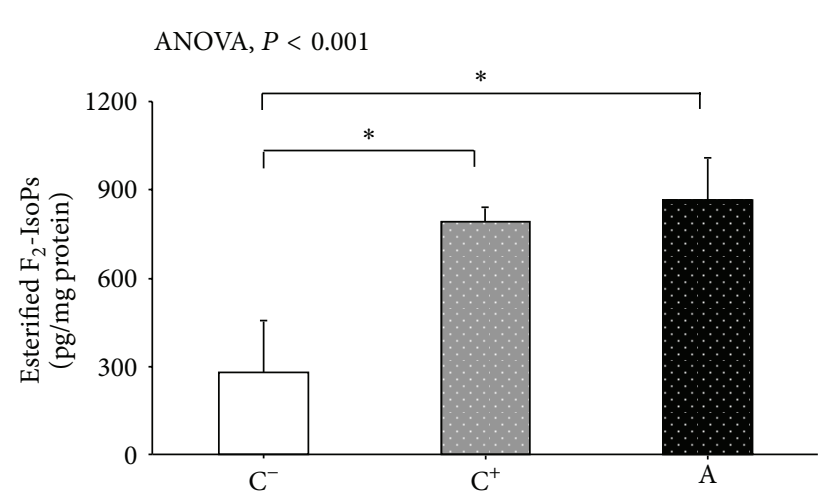

(b)

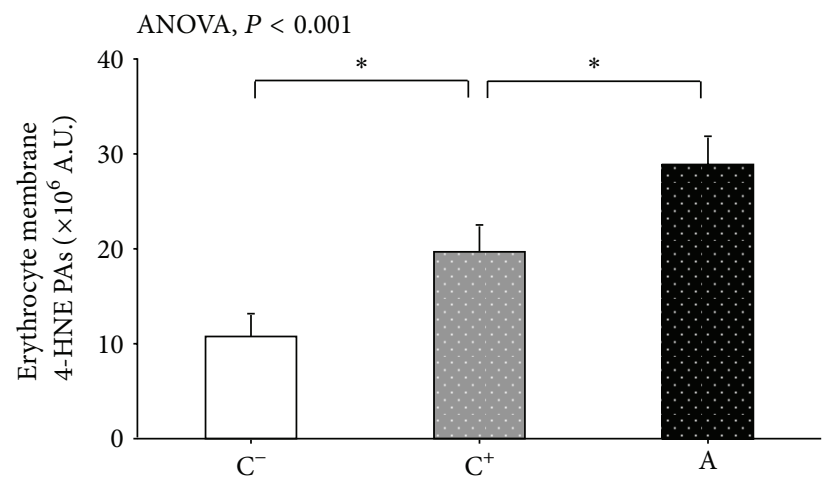

(c)

FIGURE 3: Intraerythrocyte NPBI, along with erythrocyte membrane esterified $\mathrm{F}_{2}$-IsoPs and 4-HNE protein adducts in autistic patients, subjects with nonautistic neurodevelopmental disorders (NA-NDDs), and healthy controls. NPBI was reported as $\mathrm{nmol} / \mathrm{mL}$ erythrocyte suspension, esterified $\mathrm{F}_{2}$-IsoPs as $\mathrm{pg} / \mathrm{mg}$ of erythrocyte membrane proteins, and 4-HNE protein adducts as arbitrary units. The data are expressed as means $\pm \mathrm{SD}$. Statistically significant differences are denoted by asterisks, $P<0.05$. NPBI: non protein-bound-iron; $\mathrm{F}_{2}-$ IsoPs: $\mathrm{F}_{2}$-isoprostanes; 4-HNE PAs: 4-HNE protein adducts.

linkages in membrane skeleton due either to a defective spectrin dimer-dimer interaction or a defective spectrinactin-protein $4.1 \mathrm{R}$ junctional complex. The severity of the disease is related to extent of decrease in membrane mechanical stability and resultant loss of membrane surface area.
In contrast to the healthy population, in which elliptical RBCs shapes are up to $15 \%$ of erythrocytes, a diagnosis of elliptocytosis requires that at least $25 \%$ of erythrocytes in the specimen are abnormally elliptical in shape [25]. Therefore, it can be hypothesized that autism and hereditary elliptocytosis, 


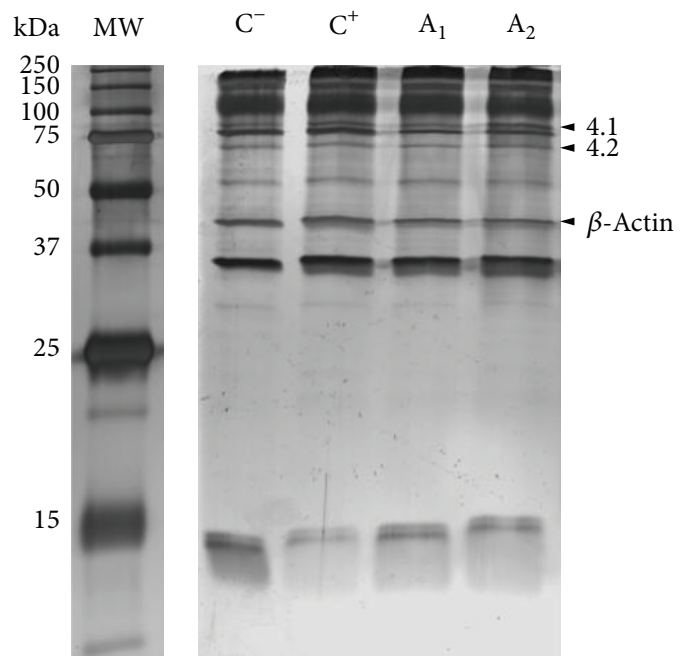

(a)

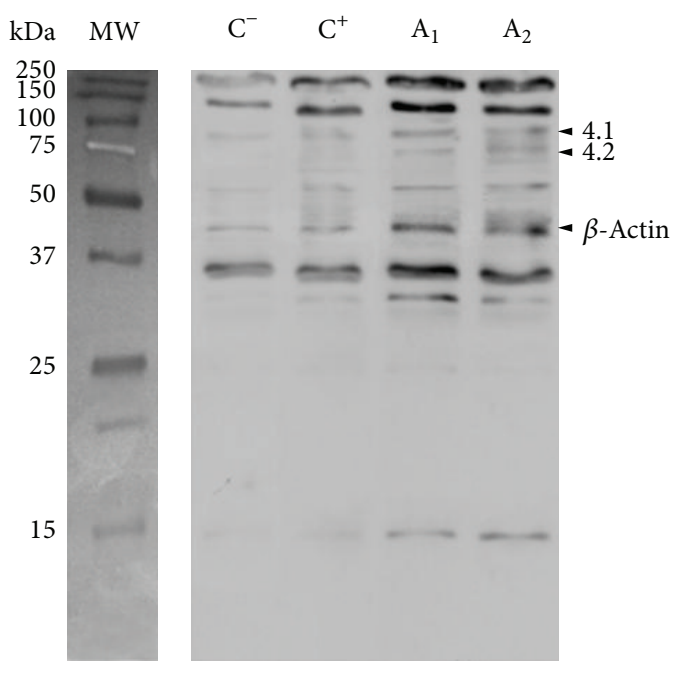

(c)

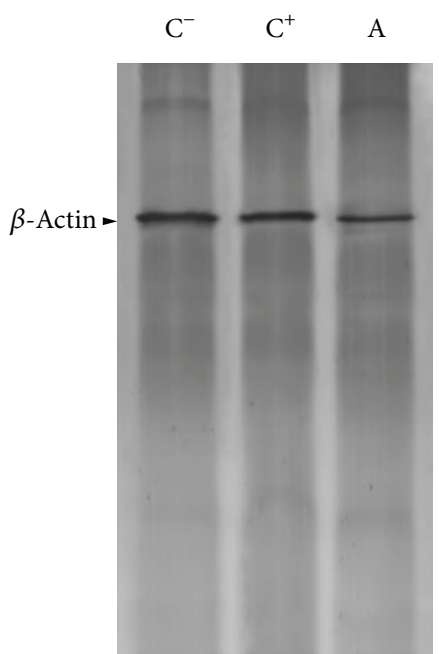

(b)

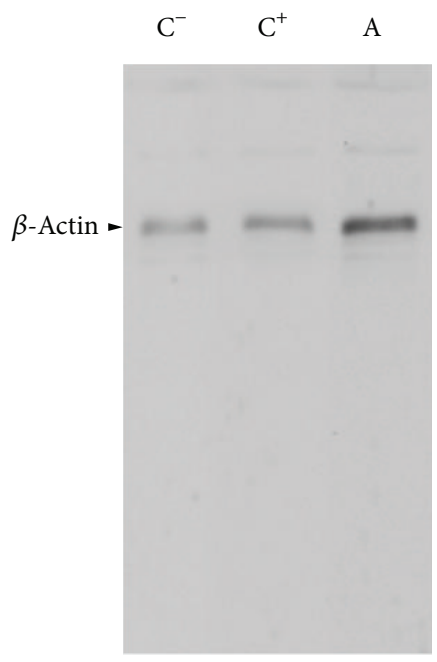

(d)

Figure 4: (a) Representative SDS-PAGE analyses of RBC ghosts (silver staining) in a healthy control subject (lane $\mathrm{C}^{-}$), a positive control subject (see text for definition; lane $\mathrm{C}^{+}$), and two autistic patients (lanes $\mathrm{A}_{1}$ and $\mathrm{A}_{2}$ ): visible reduction of intensity for $\beta$-actin in autistic patients (lanes $A_{1}$ and $A_{2}$ ) is evidenced; the electrophoretic position of bands 4.1 and 4.2 is indicated by arrowheads; (b) SDS-PAGE of immunoprecipitated $\beta$-actin (silver staining) from RBC ghosts of a healthy control subject (lane $\mathrm{C}^{-}$), a positive control individual (lane $\mathrm{C}^{+}$), and an autistic patient (lane A): visible reduction of intensity for $\beta$-actin in autistic patients (lane A); (c) immunochemical detection in the 4-HNE protein adducts in RBC ghosts: representative western blot from a healthy control subject (lane $\mathrm{C}^{-}$), a positive control individual (lane $\mathrm{C}^{+}$) and from an autistic patients (lanes $\mathrm{A}_{1}$ and $\mathrm{A}_{2}$ ). An increase in the 4-HNE PAs signal is evident in RBC ghosts from autistic patients (lanes $\mathrm{A}_{1}$ and $\mathrm{A}_{2}$ ), in particular as it concerns the $\beta$-actin band; the 4-HNE binding to bands 4.1 and 4.2 is indicated by arrowheads; and (d) immunochemical detection in the 4-HNE protein adducts in immunoprecipitated $\beta$-actin from RBC ghosts: representative western blot from a healthy control subject (lane $\mathrm{C}^{-}$), positive control individual (lane $\mathrm{C}^{+}$), and from an autistic patient (lane A): a visible increase in the 4-HNE PAs signal in $\beta$-actin from autistic patient (lane A) is evident. Molecular weight marks are indicated on the right side.

an inherited blood disorder with an estimated incidence of 3 and 5 per 10,000 in the US population, may share common physiopathological mechanisms, although neither clinical signs of hemolytic anemia nor evidence of a shortened RBCs lifespan are known in autistic patients.

The NA-NDDs group, on the other hand, shows a heterogenous variety of abnormal RBC shape changes, with only $\sim 40 \%$ of discocytes and a slightly dominance of leptocytes which appears to be just below $25 \%$ of all erythrocyte shapes in the blood samples. In this patient group, the SEM data were associated with a lower hemoglobin content and a higher degree of anisocytosis. Interestingly, we have previously described a marked ( $55 \%)$ leptocytosis in $\omega$-3 PUFAs unsupplemented girls with RTT [12], a neurodevelopmental disorder known to be the result of a single gene mutation [13]. In the near future, in our opinion, possible membrane cytoskeletal changes are to be carefully explored in this particular cause of cognitive impairment with autistic features. 
TABLE 3: Correlation matrix for elliptic erythrocyte shape, oxidative stress markers, and $\beta$-actin erythrocyte membrane content.

\begin{tabular}{|c|c|c|c|c|c|c|}
\hline & Elliptocytes & 4-HNE PAs & IE-NPBI & $\mathrm{F}_{2}$-IsoPs & $\beta$-Actin content & $\begin{array}{c}\text { 4-HNE } \beta \text {-Actin } \\
\text { adducts }\end{array}$ \\
\hline Elliptocytes & & $\begin{array}{c}0.779 \\
{[0.630-0.873]} \\
P<0.0001\end{array}$ & $\begin{array}{c}0.334 \\
{[0.045-0.572]} \\
P=0.00246\end{array}$ & $\begin{array}{c}0.588 \\
{[0.356-0.752]} \\
P<0.0001\end{array}$ & $\begin{array}{c}-0.860 \\
{[-0.921 \text { to }-0.758]} \\
P<0.0001\end{array}$ & $\begin{array}{c}0.887 \\
{[0.803-0.937]} \\
P<0.0001\end{array}$ \\
\hline 4-HNE PAs & & & $\begin{array}{c}0.397 \\
{[0.117-0.618]} \\
P=0.0068\end{array}$ & $\begin{array}{c}0.774 \\
{[0.622-0.869]} \\
P<0.0001\end{array}$ & $\begin{array}{c}-0.886 \\
{[-0.936 \text { to }-0.801]} \\
P<0.0001\end{array}$ & $\begin{array}{c}0.889 \\
{[0.806-0.938]} \\
P<0.0001\end{array}$ \\
\hline IE-NPBI & & & & $\begin{array}{c}0.415 \\
{[0.139-0.632]} \\
P=0.0045\end{array}$ & $\begin{array}{c}-0.414 \\
{[-0.631 \text { to }-0.138]} \\
P=0.0046\end{array}$ & $\begin{array}{c}0.340 \\
{[0.052-0.576]} \\
P=0.0223\end{array}$ \\
\hline $\begin{array}{l}\mathrm{F}_{2} \text {-IsoPs } \\
\beta \text {-Actin content }\end{array}$ & & & & & $\begin{array}{c}-0.628 \\
{[-0.778 \text { to }-0.410]} \\
P<0.0001\end{array}$ & $\begin{array}{c}0.579 \\
{[0.344-0.746]} \\
P<0.0001 \\
-0.960 \\
{[-0.978 \text { to }-0.929} \\
P<0.0001\end{array}$ \\
\hline $\begin{array}{l}\text { 4-HNE } \\
\beta \text {-Actin Adducts }\end{array}$ & & & & & & \\
\hline
\end{tabular}

Data are Spearman's rho correlation coefficients (in square brackets are the $95 \%$ confidence intervals for regression).

IE-NPBI: intraerythrocyte nonprotein-bound-iron; $\mathrm{F}_{2}$-IsoPs: $\mathrm{F}_{2}$-isoprostanes; 4-HNE Pas: 4-HNE protein adducts.

Although iron deficiency in about a quarter of autistic children has been recently reported, resulting in iron deficiency anemia in about $15 \%$ of the cases and related to detective iron intake [26], none of our examined patients had clinically evident anemia. Interestingly, in a series of conditions, collectively termed "neuroacanthocytoses," a distinctly abnormal erythrocyte shape (i.e., acanthocytosis) is reported to be associated with neurodegeneration and specific RBCs protein defects [27].

Genetic analyses over the past two decades have linked a full host of rare mutations to increased risk for ASDs, with a list of hundreds of autism risk loci in the human genome [28], whereas also common genetic variants are reported to affect the ASDs risk, but their individual effects appear to be modest [29]. Nevertheless, to the best of our knowledge, no genetic mutations involving the erythrocyte cytoskeleton have been previously described in autism.

4.2. Preliminary Assessment of the Relationship between $R B C$ Biology and Autistic Behavior. Estimated IQ was significantly different between the two neurodevelopmental groups (i.e., autistic versus NA-NDDs), thus suggesting that the NANDDs group was actually composed by nonautistic patients with a demonstrated cognitive impairment, although of a less severe entity, as compared with that observed in the autistic population. Therefore, it is possible that stepwise changes in red blood cell phenotype between the autistic, positive, and negative control groups may be more closely linked to IQ than autism-specific differences. As a consequence, caution is needed before stating that an elliptic RBC shape is synonymous of autism, as we indicate that the triad "RBC shape + membrane OS damage $+\beta$-actin alteration" is associated with autism rather than elliptocytosis per se.

Furthermore, during our preliminary studies, we found that patients not currently included in the NA-NDDS nor in the autistic group showing mild "autistic like features" (i.e., a few patients with anorexia nervosa (nervous anorexia), currently classified as a psychiatric illness in the group of eating disorders and two patients with juvenile schizophrenia) showed an intermediate percentage of elliptocytes between the values here reported for the NA-NDDs and the control population (our unpublished data). Therefore, a proportionality between percentage of elliptocytes in the peripheral blood and autistic features in the behavior might be present, although, at the present time, we have no definite proof for it.

4.3. RBCs Cytoskeletal Proteins: $\beta$-Actin. Erythrocyte membrane integrity is critical for maintaining the erythrocyte characteristic shape and is based on both vertical and horizontal interactions among the cytoskeletal proteins, the integral membrane proteins, and the phospholipid bilayer. Vertical interactions are based either on spectrin, ankyrin, and band 3 protein or spectrin, 4.1 protein, and glycophorin, while horizontal interactions are mainly based on spectrin, 4.1 protein and actin [30-32]. In particular, $\beta$-actin is a globular protein composed of filaments, weakly binding to the tail end of $\alpha$ and $\beta$ spectrins [33]. Our present findings evidence a deficiency in $\beta$-actin in the RBCs membranes from patients affected by classical autism, thus suggesting the coexistence of defective horizontal interaction forces in the cytoskeletal proteins of the erythrocyte membranes of these patients.

Besides the potential effects of $\beta$-actin deficiency on the membrane cytoskeletal structure of classical autistic patients, the detected $\beta$-actin alterations might be even more farranging given that emerging evidence indicated a role for $\beta$-actin in motor neuron function and axonal regeneration [34]. In particular, it has been suggested that distinct dynamics of $\mathrm{Ca}^{2+}-\beta$-actin could be a critical player in mediating the localized actin polymerization required for cellular 
constriction events mediating tissue bending, synaptic plasticity, and behavior [34, 35].

4.4. Potential Role of Exogenous or Endogenous Factor on the Erythrocyte Changes. Emerging evidence indicates that the role of environmental factors acting on genome by epigenetic regulation is relevant in autism and other neurodevelopmental diseases [36]. Moreover, a large number of toxic substances are known to cause erythrocyte damage in both experimental (i.e., phenylhydrazine and dapsone) $[14,37]$ and human settings (i.e., lead, penicillins, methyl-DOPA, and antiarrhythmics) [38], often leading to haemolytic anemias through different mechanisms. In our patients no clues exist for a specific exogenous molecule potentially causing an increased prevalence of elliptocytosis, and no evidence of haemolytic anemias was detectable. To date, no specific gene mutations involving the cytoskeletal components of the erythrocyte membrane have been demonstrate. Therefore, the demonstrated alteration of $\beta$-actin in our autistic patients appears to be the effect of a posttranslational modification, rather than the result of a specific gene mutation in the progenitor erythrocyte cells.

4.5. Oxidative Stress of the Erythrocyte Membrane. A link between oxidative stress and ASD has been previously reported by several authors [39-41]. Over the last few years, our team has demonstrated that OS is a key player in modulating the genotype-phenotype expression in RTT [20, 42-46].

Moreover, in previous reports, a correlation between OS and ASD has been widely explored by measuring different molecules, possibly coming from oxidative pathway as metabolic biomarkers of OS, in biological fluids [10, 39].

In autistic patients, the immunochemical detection of 4-HNE PAs indicate that oxidative events are ongoing in the RBCs of autistic patients and individuals with several neurodevelopmental disorders without autistic features. The reduction in membrane $\beta$-actin appears to be inversely related to the levels of 4 -HNE $\beta$-AAs, thus indicating that the apparently reduced protein expression in the erythrocyte membranes from autistic patients is rather the consequence of a peculiar posttranscriptional modification linked to lipid peroxidation than the results of a reduced protein synthesis. This event, triggered by NPBI as a prooxidant factor [47], produces several compounds of degradation, 4-HNE among them. This highly reactive aldehyde can covalently bind proteins, phospholipids, and DNA; in particular, 4-HNE reacts readily with nucleophilic groups of amino acidic side chains, and its covalent attachment to proteins lead to alteration in their structure and biological activity [48]. Depending on its concentration and location, 4-HNE may be therefore considered as a "second toxic messenger," which disseminates and augments initial free radical events.

Although a protective physiological role for OS-for instance oxidative shielding-has been recently underlined [49], there is little doubt that an oxidized protein is a damaged molecule with a likely reduced function. Thus, within the red cell membrane environment, oxidized proteins contribute to alter the phospholipid bilayer integrity and weaken the membrane mechanical properties, including a loss of the membrane fluidity as already reported in autism [6]. The report of significant alterations in the fatty acid profiles in individuals with ASDs in erythrocyte membrane [50] appears to be in line with our morphological and biochemical observations. Although an increased lipid peroxidation in the erythrocyte membrane from autistic patients has been suggested by using a thiobarbituric acid reactive substances (TBARs) assay kit [11], to date no further information on the in situ lipid and protein membrane damage is available. Our findings of increased erythrocyte membrane $\mathrm{F}_{2}$-IsoPs, as measured by a specific spectrometric method, and increased membrane 4-HNE-PAs reveal an increased arachidonic acid peroxidation, with a subsequent protein posttranslational modification in the erythrocyte membrane of patients with classical autism and NA-NDDs.

\section{Conclusions}

Our findings indicate the presence of an unrecognized triad combination of erythrocyte shape abnormalities, erythrocyte membrane oxidative damage, and $\beta$-actin alterations in classical autism and provides new biological markers in the diagnostic workup of ASDs. At least two unsolved questions are generated by our observations. Firstly, the specificity of our findings to autistic disorders is unknown to date and needs to be further explored. Secondly, the relationship between the abnormal erythrocyte shape and the neurological development in autistic children needs to be further investigated. The reported alteration in erythrocyte shape for classical autism could be theoretically translatable into a routine technology, such as fluorescence-activated cell sorting (FACS) either testing the volume or the morphological complexity of cells, in addition to the membrane fluidity.

In conclusion, our data shed new light on the concept of OS as a key factor in the pathogenesis of neurodevelopmental disorders. Interestingly, our data indicate that erythrocyte shape, either due to a defective RBC cytoskeletal scaffold or being the consequence of an oxidative cell damage, could be considered as a new potential physical biomarker for neurodevelopmental disorders.

\section{Conflict of Interests}

The authors declare that they have no conflict of interest.

\section{Authors' Contribution}

Lucia Ciccoli and Claudio De Felice contributed equally to this work.

\section{Acknowledgments}

The present research project has been funded by the Tuscany Region (Bando Salute 2009, "Antioxidants ( $\omega$-3 Polyunsaturated Fatty Acids, lipoic acid) supplementation in Rett syndrome: A novel approach to therapy"), Italy. The authors sincerely thank Drs. Pierluigi Tosi, Silvia Briani, and Roberta Croci from the Administrative Direction of the Azienda Ospedaliera Senese for continued support to their studies and the Azienda Ospedaliera Senese for prior 
purchasing of the gas spectrometry instrumentation. We thank Roberto Faleri (Central Medical Library, University of Siena, Siena, Italy) for online bibliographic assistance. The authors sincerely thank the professional singer Matteo Setti (http://www.matteosetti.com/) for having serendipitously triggered the scientific studies on hypoxia-related oxidative stress in Rett girls and autistic children, as well as his many charity concerts and continued interest and support in the scientific aspects of our research.

\section{References}

[1] M. R. Herbert, "Contributions of the environment and environmentally vulnerable physiology to autism spectrum disorders," Current Opinion in Neurology, vol. 23, no. 2, pp. 103-110, 2010.

[2] C. Testa, F. Nuti, J. Hayek et al., "Di-(2-ethylhexyl) phthalate and autism spectrum disorders," The American Society For Neurochemistry, vol. 4, no. 4, pp. 223-229, 2012.

[3] E. Fombonne, "Is there an epidemic of autism?" Pediatrics, vol. 107, no. 2, pp. 411-413, 2001.

[4] J. Baio, "Prevalence of Autism spectrum disorders: autism and developmental disabilities monitoring network, 14 Sites, United States, 2008," Morbidity and Mortality Weekly Report, vol. 61, no. 3, pp. 1-19, 2012.

[5] K. Weintraub, “The prevalence puzzle: autism counts," Nature, vol. 479 , no. 7371, pp. 22-24, 2011.

[6] A. Chauhan and V. Chauhan, "Oxidative stress in autism," Pathophysiology, vol. 13, no. 3, pp. 171-181, 2006.

[7] A. Pecorelli, S. Leoncini, C. De Felice et al., "Non-proteinbound iron and 4-hydroxynonenal protein adducts in classic autism," Brain and Development, vol. 35, no. 2, pp. 146-154, 2013.

[8] Frustaci, M. Neri, A. Cesario et al., "Oxidative stress-related biomarkers in autism: systematic review and meta-analyses," Free Radical Biology and Medicine, vol. 52, no. 10, pp. 2128-2141, 2012.

[9] L. Palmieri and A. M. Persico, "Mitochondrial dysfunction in autism spectrum disorders: cause or effect?" Biochimica et Biophysica Acta, vol. 1797, no. 6-7, pp. 1130-1137, 2010.

[10] A. Chauhan, T. Audhya, and V. Chauhan, "Brain region-specific glutathione redox imbalance in autism," Neurochemical Research, vol. 37, no. 8, pp. 1681-1689, 2012.

[11] A. Ghezzo, P. Visconti, P. M. Abruzzo et al., "Oxidative stress and erythrocyte membrane alterations in children with autism: correlation with clinical features," PLoS ONE, vol. 8, no. 6, Article ID e66418, 2013.

[12] L. Ciccoli, C. De Felice, E. Paccagnini et al., "Morphological changes and oxidative damage in Rett Syndrome erythrocytes," Biochimica et Biophysica Acta, vol. 1820, no. 4, pp. 511-520, 2012.

[13] M. Chahrour and H. Y. Zoghbi, "The story of rett syndrome: from clinic to neurobiology," Neuron, vol. 56, no. 3, pp. 422-437, 2007.

[14] L. Ciccoli, C. Signorini, C. Alessandrini, M. Ferrali, and M. Comporti, "Iron release, lipid peroxidation, and morphological alterations of erythrocytes exposed to acrolein and phenylhydrazine," Experimental and Molecular Pathology, vol. 60, no. 2, pp. 108-118, 1994.

[15] M. A. Srour, Y. Y. Bilto, M. Juma, and M. R. Irhimeh, "Exposure of human erythrocytes to oxygen radicals causes loss of deformability, increased osmotic fragility, lipid peroxidation and protein degradation," Clinical Hemorheology and Microcirculation, vol. 23, no. 1, pp. 13-21, 2000.
[16] G. Lucantoni, D. Pietraforte, P. Matarrese et al., “The red blood cell as a biosensor for monitoring oxidative imbalance in chronic obstructive pulmonary disease: an ex vivo and in vitro study," Antioxidants and Redox Signaling, vol. 8, no. 7-8, pp. 11711182, 2006.

[17] A. László, Z. Novák, I. Szőllősi-Varga, Q. Hai du, Á. Vetró, and A. Kovács, "Blood lipid peroxidation, antioxidant enzyme activities and hemorheological changes in autistic children," Ideggyogy Szemle, vol. 66, no. 1-2, pp. 23-28, 2013.

[18] E. Schopler, R. J. Reichler, R. F. DeVellis, and K. Daly, “Toward objective classification of childhood autism: childhood autism rating scale (CARS)," Journal of Autism and Developmental Disorders, vol. 10, no. 1, pp. 91-103, 1980.

[19] J. Walters and P. Garrity, "Performance evaluation of the sysmex XE-2100 hematology analyzer," Laboratory Hematology, vol. 6, pp. 83-92, 2000.

[20] C. De Felice, L. Ciccoli, S. Leoncini et al., "Systemic oxidative stress in classic Rett syndrome," Free Radical Biology and Medicine, vol. 47, no. 4, pp. 440-448, 2009.

[21] J. T. Dodge, C. Mitchell, and D. J. Hanahan, “The preparation and chemical characteristics of hemoglobin-free ghosts of human erythrocytes," Archives of Biochemistry and Biophysics, vol. 100, no. 1, pp. 119-130, 1963.

[22] U. K. Laemmli, "Cleavage of structural proteins during the assembly of the head of bacteriophage T4," Nature, vol. 227, no. 5259, pp. 680-685, 1970.

[23] M. Bessis, Réinterprétation des Frottis Sanguins, Springer, Berlin, Germany, 1st edition, 1977.

[24] S. Chien, "Red cell deformability and its relevance to blood flow," Annual Review of Physiology, vol. 49, pp. 177-192, 1987.

[25] W. Barcellini, P. Bianchi, E. Fermo et al., "Hereditary red cell membrane defects: diagnostic and clinical aspects," Blood Transfusion, vol. 9, no. 3, pp. 274-277, 2011.

[26] S. Hergüner, F. M. Keleşoğlu, C. Tanıdır, and M. Cöpür, "Ferritin and iron levels in children with autistic disorder," European Journal of Pediatrics, vol. 171, no. 1, pp. 143-146, 2012.

[27] R. Prohaska, O. C. M. Sibon, D. D. Rudnicki et al., "Brain, blood, and iron: perspectives on the roles of erythrocytes and iron in neurodegeneration," Neurobiology of Disease, vol. 46, no. 3, pp. 607-624, 2012.

[28] S. J. Sanders, A. G. Ercan-Sencicek, V. Hus et al., "Multiple recurrent de novo CNVs, including duplications of the 7q11.23 Williams syndrome region, are strongly associated with autism," Neuron, vol. 70, no. 5, pp. 863-885, 2011.

[29] R. Anney, L. Klei, D. Pinto et al., "Individual common variants exert weak effects on risk for Autism Spectrum Disorders," Human Molecular Genetics, vol. 21, no. 21, pp. 4781-4792, 2012.

[30] S. Rocha, I. Rebelo, E. Costa et al., "Protein deficiency balance as a predictor of clinical outcome in hereditary spherocytosis," European Journal of Haematology, vol. 74, no. 5, pp. 374-380, 2005.

[31] W. Nunomura and Y. Takakuwa, "Regulation of protein 4.1R interactions with membrane proteins by $\mathrm{Ca} 2+$ and calmodulin," Frontiers in Bioscience, vol. 11, no. 2, pp. 1522-1539, 2006.

[32] P. G. Gallagher and P. Jarolim, "Red blood cell membrane disorders," in Hematology: Basic Principles and Practice, R. Hoffman, B. Furie, E. J. Benz, P. McGlave, L. E. Silberstein, and S. J. Shattil, Eds., pp. 623-643, Churchill Livingstone, Philadelphia, Pa, USA, 2009.

[33] X. An, G. Debnath, X. Guo et al., "Identification and functional characterization of protein $4.1 \mathrm{R}$ and actin-binding sites in 
erythrocyte $\beta$ spectrin: regulation of the interactions by phosphatidylinositol-4,5-bisphosphate," Biochemistry, vol. 44, no. 31, pp. 10681-10688, 2005.

[34] T. R. Cheever, E. A. Olson, and J. M. Ervasti, "Axonal regeneration and neuronal function are preserved in motor neurons lacking beta-actin In Vivo," PLoS ONE, vol. 6, no. 3, Article ID e17768, 2011.

[35] T. R. Cheever and J. M. Ervasti, "Actin isoforms in neuronal development and function," in International Review of Cell and Molecular Biology, K. W. Jeon, Ed., pp. 157-213, Elsevier/Academic Press, 2013.

[36] K. Miyake, T. Hirasawa, T. Koide, and T. Kubota, "Epigenetics in autism and other neurodevelopmental diseases," Advances in Experimental Medicine and Biology, vol. 724, pp. 91-98, 2012.

[37] L. Ciccoli, M. Ferrali, V. Rossi, C. Signorini, C. Alessandrini, and M. Comporti, "Hemolytic drugs aniline and dapsone induce iron release in erythrocytes and increase the free iron pool in spleen and liver," Toxicology Letters, vol. 110, no. 1-2, pp. 57-66, 1999.

[38] V. Kumar, A. K. Abbas, N. Fausto, and J. C. Aster, Robbins and Cotran Pathologic Basis of Disease, Elsevier, 2010.

[39] S. J. James, P. Cutler, S. Melnyk et al., "Metabolic biomarkers of increased oxidative stress and impaired methylation capacity in children with autism," The American Journal of Clinical Nutrition, vol. 80, no. 6, pp. 1611-1617, 2004.

[40] K.-A. Villagonzalo, S. Dodd, O. Dean, K. Gray, B. Tonge, and M. Berk, "Oxidative pathways as a drug target for the treatment of autism," Expert Opinion on Therapeutic Targets, vol. 14, no. 12, pp. 1301-1310, 2010.

[41] E. M. Sajdel-Sulkowska, M. Xu, W. McGinnis, and N. Koibuchi, "Brain region-specific changes in oxidative stress and neurotrophin levels in autism spectrum disorders (ASD)," Cerebellum, vol. 10, no. 1, pp. 43-48, 2011.

[42] A. Pecorelli, L. Ciccoli, C. Signorini et al., "Increased levels of 4HNE-protein plasma adducts in Rett syndrome," Clinical Biochemistry, vol. 44, no. 5-6, pp. 368-371, 2011.

[43] S. Leoncini, C. de Felice, C. Signorini et al., "Oxidative stress in Rett syndrome: natural history, genotype, and variants," Redox Report, vol. 16, no. 4, pp. 145-153, 2011.

[44] C. Signorini, C. De Felice, S. Leoncini et al., "F4-neuroprostanes mediate neurological severity in Rett syndrome," Clinica Chimica Acta, vol. 412, no. 15-16, pp. 1399-1406, 2011.

[45] C. De Felice, C. Signorini, S. Leoncini et al., "The role of oxidative stress in Rett syndrome: an overview," Annals of the New York Academy of Sciences, vol. 1259, no. 1, pp. 121-135, 2012.

[46] E. Grillo, C. Lo Rizzo, L. Bianciardi et al., "Revealing the complexity of a monogenic disease: rett syndrome exome sequencing," PLoS ONE, vol. 8, no. 2, Article ID e56599, 2013.

[47] L. Ciccoli, S. Leoncini, C. Signorini, and M. Comporti, "Iron and erythrocytes: physiological and pathophysiological aspects," in Oxidant in Biology, G. Valacchi and P. Davis, Eds., pp. 167-181, Springer, Heidelberg, Germany, 2008.

[48] E. E. Dubinina and V. A. Dadali, "Role of 4-hydroxy-trans-2nonenal in cell functions," Biochemistry, vol. 75, no. 9, pp. 10691087, 2010.

[49] R. K. Naviaux, "Oxidative shielding or oxidative stress?" Journal of Pharmacology and Experimental Therapeutics, vol. 342, no. 3, pp. 608-618, 2012.

[50] C. M. Brown and D. W. Austin, "Autistic disorder and phospholipids: a review," Prostaglandins Leukotrienes and Essential Fatty Acids, vol. 84, no. 1-2, pp. 25-30, 2011. 


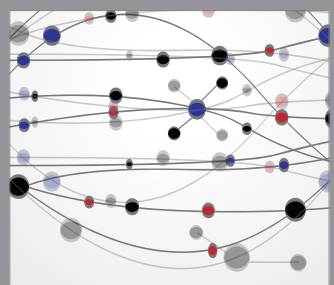

The Scientific World Journal
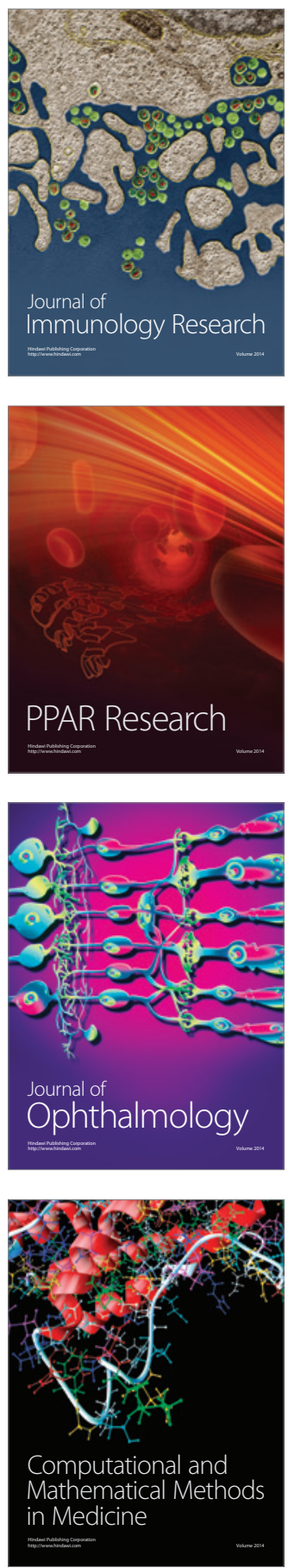

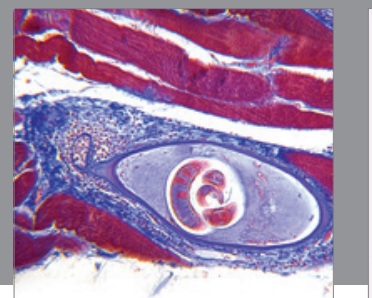

Gastroenterology

Research and Practice
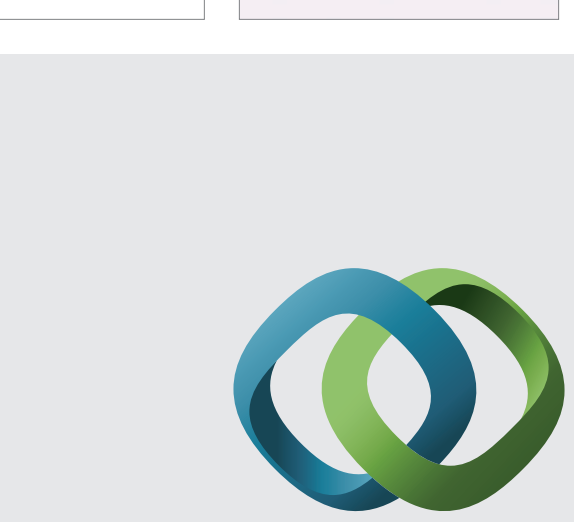

\section{Hindawi}

Submit your manuscripts at

http://www.hindawi.com
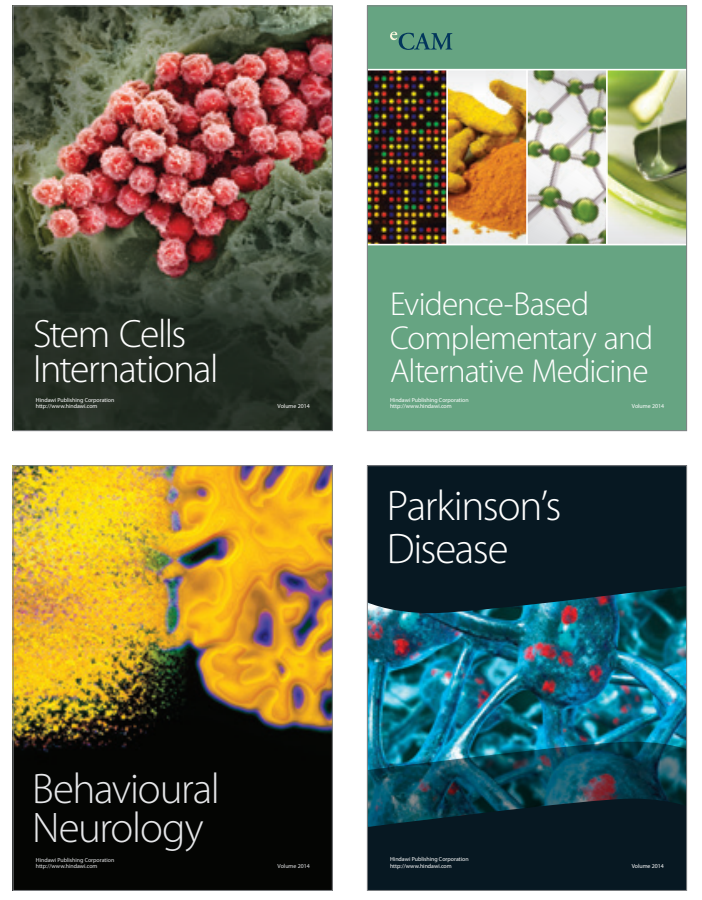
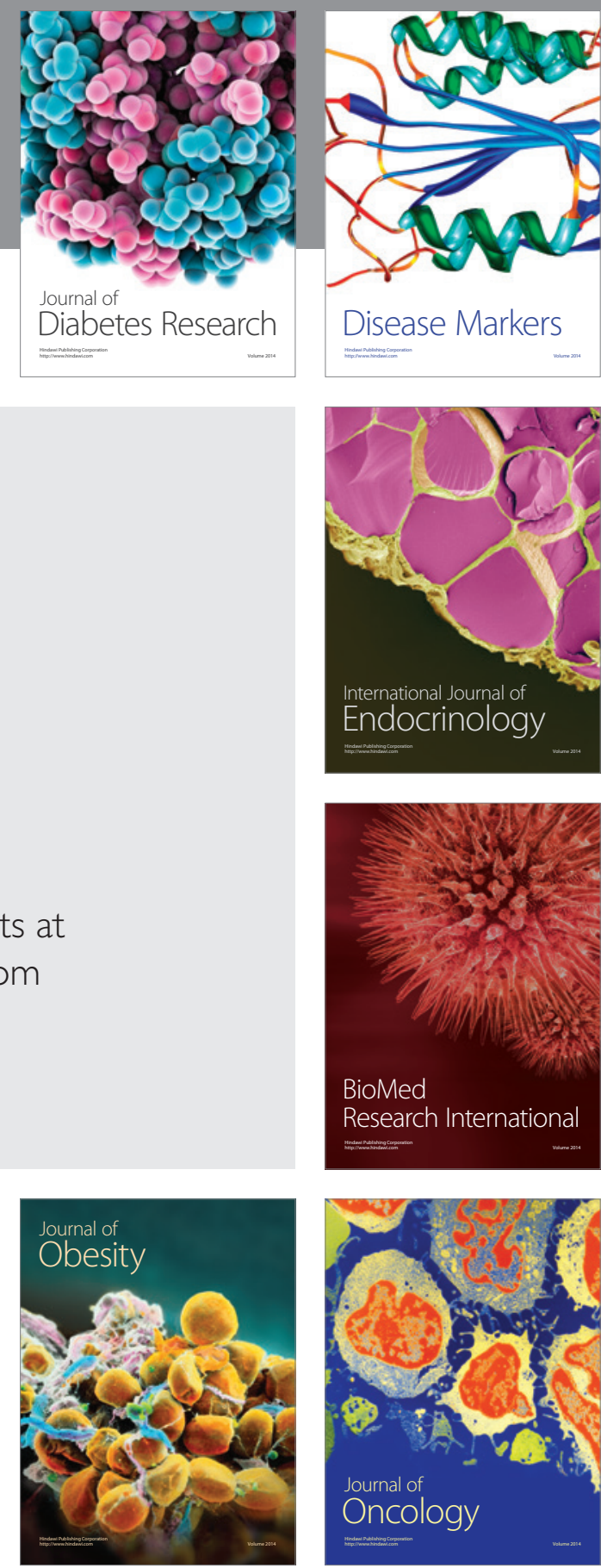

Disease Markers
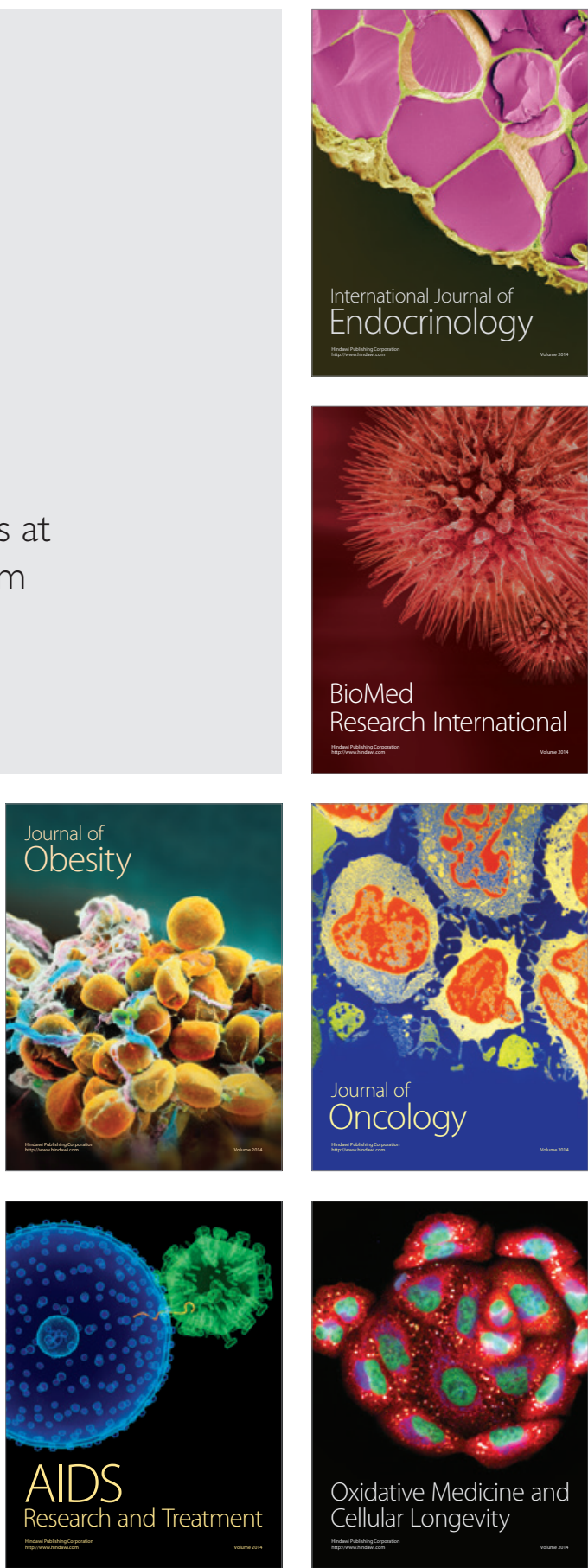